\title{
Residual Finiteness of Surface Groups via Tessellations
}

\author{
L. M. Lopez \\ Department of Information Science, Tokyo Institute of Technology, \\ Oh-Okayama, Meguro-ku, Tokyo 152, Japan \\ lopez@titisha.is.titech.ac.jp
}

\begin{abstract}
We give an alternative proof to a theorem by P. Scott that surface groups have the locally extended residually finite (LERF) property, using combinatorial properties of tilings of the hyperbolic plane.
\end{abstract}

\section{Introduction}

A group $G$ is said to be locally extended residually finite (LERF) if, for any finitely generated subgroup $S$ of $G$ and any element $g$ of $G-S$, there exists a finite index subgroup $H$ of $G$ such that $S \subset H$ and $g \notin H$. When we take $S=\{1\}$ we obtain the definition of residual finiteness.

For example, Fuchsian groups are LERF: this is a theorem due to Scott; see [5] and [6] (and [2] for another proof). It seems that there are also works by R. Gitik and by G. A. Niblo which generalize Scott's theorem, but the author has not seen them.

Since any Fuchsian group contains a closed orientable surface fundamental group as a finite index subgroup, and a group having an LERF subgroup of finite index is itself LERF we concentrate on the latter groups. Namely, we prove:

Theorem A. Let $F$ be a closed orientable surface. Then $\pi_{1}(F)$ is $L E R F$.

The approach for constructing subgroups is the same as the one used by Scott: we use the natural correspondence between subgroups of the fundamental group, and covering spaces. However, the key point of the proof presented here relies on a combinatorial argument, rather than on a geometric-analytic one.

The motivation for this work can be stated as follows: tessellations allow us to visualize algebraic properties of groups, as in Chapter 2 of [3]. The question 
addressed here is the converse one: from vizualizations of groups by tessellations what kind of algebraic properties can be derived? Tessellations are of independent interest and raise many interesting problems in combinatorics, as in [1] (see also the bibliography listed at the end of [1]).

\section{Proof of Theorem $A$}

If $X$ is a topological subspace of some space, $\operatorname{int}(X)$ means the topological interior of $X$. The boundary of a manifold $X$ is denoted by $\partial X$. A surface is a connected orientable two-dimensional manifold.

Let $m$ be an integer greater than 1 . We consider first a regular quadrilateral in the hyperbolic plane $\mathbb{H}^{2}$, with angle $2 \pi / 4(2 m-1)$ (Fig. 1); this is possible since $m>1$.

We consider the "stripe" formed by $2 m-1$ copies of this quadrilateral, identified along their sides labeled $c_{i}$ and $c_{i}^{\prime}$ (Fig. 2). We label and orient the remaining sides as in Fig. 2, and obtain a $4 m$-sided polygon.

Identifying the sides according to these labelings and orientations gives a closed orientable surface, whose Euler characteristic is easy to calculate: one vertex, $2 \mathrm{~m}$ edges, and one face. Hence, $\chi(F)=2-2 m$ and $F$ is a closed surface of genus $m$. The hyperbolic metric on each quadrilateral extends to one on $F$; the only point to check is that the sum of the angles around the vertex is $2 \pi$. However, there are $2 m-1$ quadrilaterals, hence $4 \times(2 m-1)$ angles of measure $2 \pi / 4(2 m-1)$ each.

So, when looking at the universal covering of $F$, which, according to the above,

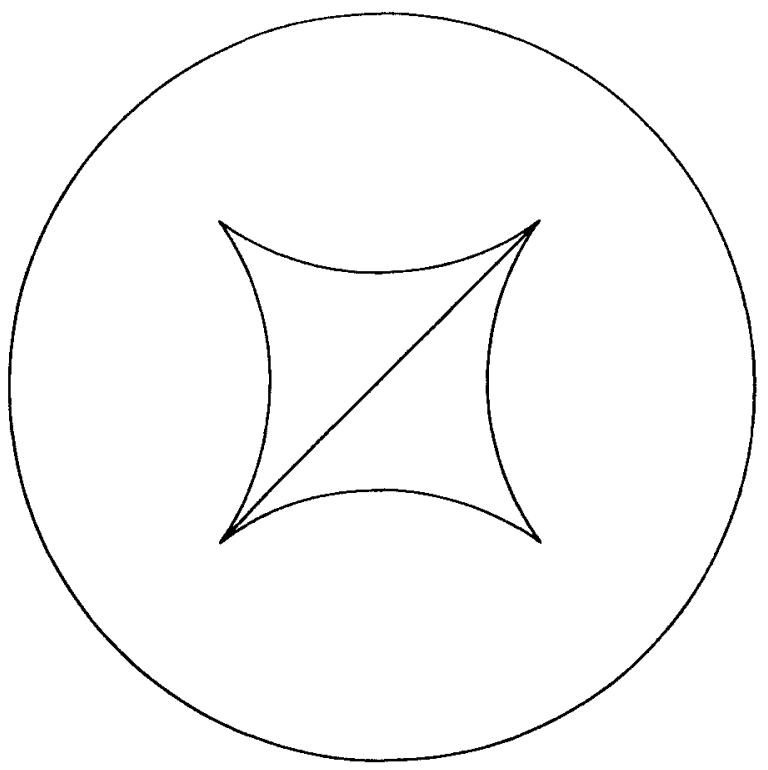

Fig. 1. A regular quadrilateral in the hyperbolic plane $\mathbb{H}^{2}$. 


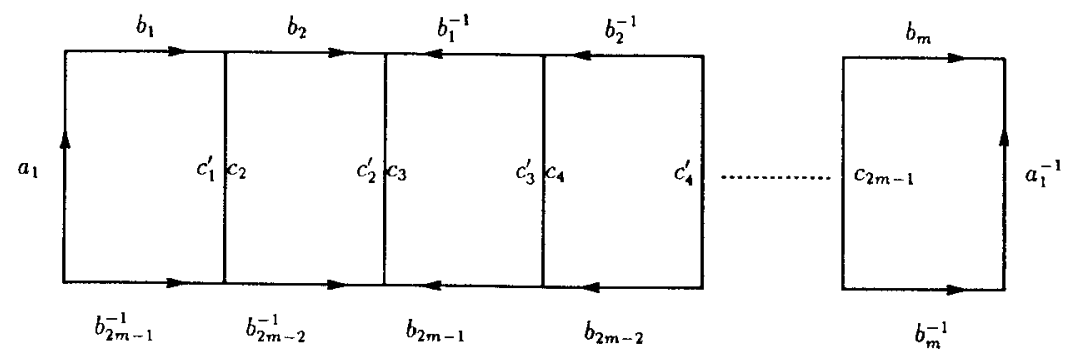

Fig. 2. The "stripe" formed by $2 m-1$ copies of the quadrilaterals.

can be identified with $\mathbb{H}^{2}$, we see a tessellation of $\mathbb{H}^{2}$ by regular quadrilaterals. We choose a diagonal for one quadrilateral (see Fig. 1), and consider one of the two triangles thus formed. The group of hyperbolic motions generated by reflections about its sides is a hyperbolic triangle group (denoted by $T(4(2 m-1)$, $4(2 m-1), 2(2 m-1))$; see [4]). There is a group of hyperbolic motions associated with tessellation by the quadrilaterals: it is the index 2 subgroup of the above group, formed by the orientation-preserving motions. We call this subgroup $G_{m}$.

By classical covering space theory we get that, once a base point has been fixed in $\mathrm{H}^{2}$, the fundamental group of any of its quotient surfaces by subgroups of $G_{m}$ can be identified with a group of hyperbolic motions. For example, $\pi_{1}(F)$ can be seen as a subgroup of $G_{m}$ of index $2 m-1$.

We now begin the proof of Theorem $\mathrm{A}$.

Let $S$ be a finitely generated subgroup of $\pi_{1}(F)$ and let $s_{0}$ be an element of it not in $S$. We denote by $s_{1}, \ldots, s_{n}$ a system of generators for $S$, and consider the quotient of $\mathbb{H}^{2}$ by the action of $S$. It is a surface without boundary, since it is a covering space of $F$, and we call it $\mathscr{S}$. By definition of $G_{m}$ there exist $n+1$ quadrilaterals containing the translates of $\mathbb{L}^{2}{ }^{2}$ 's base point by the $s_{i}$ 's. We join, on $\mathrm{H}^{2}$, these translates with the base point, with paths avoiding the vertices of the quadrilaterals, and consider the (finite) set of quadrilaterals that they visit. We call $\mathscr{U}_{1}$ the connected subset of $\mathbb{H}^{2}$ formed by the set of all translates of these quadrilaterals through the action of $S$. So $\mathscr{U}_{1}$ is an infinite union of quadrilaterals, but its quotient by $S$, called $\mathscr{U}^{\prime}$, is a finite one and subset of $\mathscr{S}$. Because of our choice for the paths, int $\left(\mathscr{U}_{1}\right)$ is connected.

The unions of quadrilaterals $\mathscr{U}^{\prime}$ and $\mathscr{U}_{1}$ may not be surfaces; if this happens it is only at a finite number of points, all being vertices. However, we can define the notion of boundary component for such spaces: we define it as a simple curve obtained as a union of free edges.

Since the number of boundary components of ' $\mathcal{U}^{\prime}$ is necessarily finite (for ' $\mathcal{U}^{\prime}$ is a finite union of quadrilaterals), the number of boundary components of $\mathscr{U}_{1}$ is also finite, up to the action of $S$.

The bounded boundary components are simple loops in $\mathrm{H}^{2}$ and hence bound disks in it, and since these loops are formed as unions of free edges of $\mathscr{U}_{1}$ these disks are unions of quadrilaterals. We add these disks and their translates to $\mathscr{U}_{1}$ and call the resulting space $\mathscr{U}_{2}$. The important fact is that $\mathscr{U}_{2}$ contains no more 


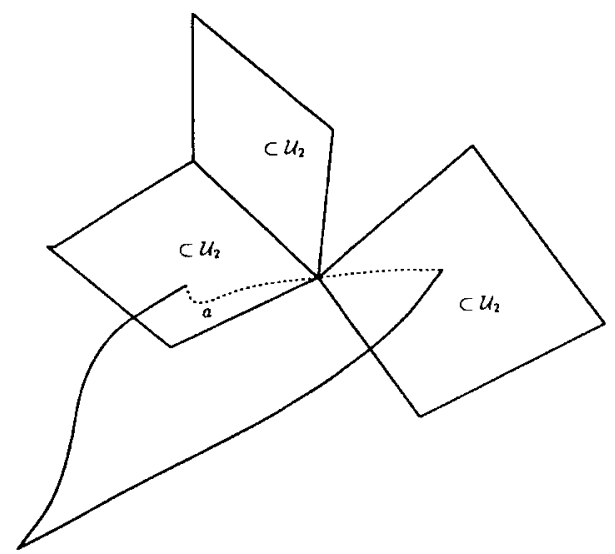

Fig. 3. The vertex in $\mathscr{U}^{\prime \prime}$ shared by two quadrilaterals.

bounded boundary components. Its quotient by the action of $S$ is finite, and we call it $\mathscr{U}^{\prime \prime}$.

Claim 1. $\mathscr{U}^{\prime \prime}$ is a surface.

Proof. Suppose not, then there exists a vertex in $\mathscr{U}^{\prime \prime}$, hence in $\mathscr{U}_{2}$, such that it is shared by (at least) two quadrilaterals, but is not an ordinary boundary point (Fig. 3).

Since $\operatorname{int}\left(\mathscr{U}_{2}\right)$ is connected there exist two paths in it joining the origin to these two quadrilaterals. Transform these paths into a loop by joining their ends with a little arc $a$ which passes through the vertex. This loop bounds a disk, and because there are free edges of $\mathscr{U}_{2}$ meeting the interior of the disk there must exist some boundary component of $\mathscr{U}_{2}$, necessarily a bounded one, contained in the disk. This contradicts the fact that $\mathscr{U}_{2}$ possesses no more bounded boundary components.

Let $x$ be a vertex in a surface obtained as a union of quadrilaterals, a quotient of $\mathbb{H}^{2}$ by a subgroup of $G_{m}$. We define the degree of $x, d(x)$, as the number of quadrilaterals in this suface having $x$ as a vertex. Hence, $1 \leq d(x) \leq 4(2 m-1)$.

We wish to apply the following proposition, whose proof is postponed to Section 3 , to the surface $\mathscr{U}^{\prime \prime}$ :

Proposition 1. Let $\mathscr{U}$ be a surface consisting of a finite union of quadrilaterals. Let $m$ be an integer greater than 1. Suppose that:

(i) the degree of any vertex lying in $\mathscr{U}-\partial \mathscr{U}$ is $k=4(2 m-1)$, and

(ii) any vertex lying in $\partial \mathscr{U}$ has degree less than $k-1$, 
then there exists a closed surface consisting of a finite union of quadrilaterals $\overline{\mathcal{U}}$, containing $\mathscr{U}$, such that the degree of any vertex is $k$.

So it suffices to check two conditions:

1. The degree of any vertex in $\mathscr{S}$ is equal to $4(2 m-1)$.

2. The degree of any vertex on the boundary of $\mathscr{U}^{\prime \prime}$ is less than $k-1$.

The former follows from the fact that $\mathscr{S}$ is a covering space of $F$, as for the latter it may not be satisfied by $\mathscr{U}^{\prime \prime}$. So we modify $\mathscr{U}^{\prime \prime}$ as follows.

We define the complexity $c(\mathscr{U})$ of a surface $\mathscr{U}$ consisting of a finite union of quadrilaterals and contained in $\mathscr{S}$ as the pair of integers:

$$
\text { (number of free edges of } \mathscr{U}, \sum d(x) \text { ), }
$$

where $x$ runs through all the vertices of the boundary components of $\mathscr{U}$. We order complexities with the lexicographic order.

We prove:

Claim 2. Let $x_{0}$ be a vertex on a boundary component of $U$ such that $d\left(x_{0}\right)=k-1$, and let $Q$ be the only quadrilateral in $\mathscr{S}$ having $x_{0}$ as a vertex and whose interior misses $\mathscr{U}$. Then either of the following occurs:

1. $\mathscr{U} \cup Q$ is not a surface at some vertex of $Q$.

2. $c(\mathscr{U} \cup Q)<c(\mathscr{U})$.

Proof. $Q$ meets $\mathscr{U}$ along at least two edges. If it is three or four, then the number of free edges for $\mathscr{U} \cup Q$ is less than for $\mathscr{U}$, and $c(\mathscr{U} \cup Q)<c(\mathscr{U})$. So we assume from now on that the number of edges common to $\mathscr{U}$ and $Q$ is just two. Notice that in this case the number of free edges remains the same for $\mathscr{U} \cup Q$ and $\mathscr{U}$.

There are two cases: $Q$ meets $\mathscr{U}$ along three vertices or four vertices. In the latter case $\mathscr{U} \cup Q$ is not a surface at a vertex $x$ of $Q$. In the former case the result is a surface and we can calculate the total degree of the boundary vertices as follows: in $\mathscr{U} \cup Q$ the vertex $x_{0}$ of degree $k-1$ in $\mathscr{U}$ has disappeared from the boundary, while one of degree 1 has appeared; the two remaining vertices of $\mathscr{U} \cap Q$ have seen their degrees increased by 1 each. So considering the total degree before and after adding $Q$ to $\mathscr{U}$ we see that it has decreased by $k-4$. Since $k>4$ we are done.

In the case $\mathscr{U} \cup Q$ is not a surface we can construct a bounded boundary component as in Claim 1's proof, since int $(\mathscr{U} \cup Q)$ is connected. We cap off bounded boundary components with disks, as in Claim 1, and get a new surface $\mathscr{V}$ in $\mathscr{S}$. The number of free boundary edges for $\mathscr{V}$ has of course decreased with respect to that of $\mathscr{U}$, since boundary components capped off with disks disappear. So $c(\mathscr{V})$ is smaller than $c(\mathscr{U})$. 
This means that by applying Claim 2 inductively to $\mathscr{U}^{\prime \prime}$ and capping off bounded boundary components with disks as above, we will eventually obtain a finite surface in $\mathscr{P}, \mathscr{U}^{(p)}$, either closed or having no more vertices of degree $k-1$ on its boundary.

If $\mathscr{U}^{(p)}$ is not a closed surface, by Proposition 1 we get a closed one, obtained as a finite union of quadrilaterals $\overline{\mathscr{U}^{(p)}}$ such that:

1. $\overline{\mathscr{U}^{(p)}}$ is a surface, quotient of $\mathbb{M}^{2}$ by the action of a subgroup of $G_{m}$ because all degrees at vertices are equal to $k$.

2. $\overline{\mathscr{U}}^{(p)}$ contains $\mathscr{U}^{\prime}$ because it contains $\mathscr{U}^{(p)}$.

3. $\pi_{1}\left(\overline{\mathscr{U}^{(p)}}\right)$ contains $\mathscr{S}$ but not $s_{0}$, since $\mathscr{U}^{\prime} \subset \overline{\mathscr{U}^{(p)}}$.

There is a finite index subgroup $H$ in $G_{m}$ corresponding to the universal covering $\mathbb{M}^{2} \rightarrow \overline{\mathscr{U}^{(p)}}$. The subgroup $H \cap \pi_{1}(F)$ has the required properties with respect to $S$ and $s_{0}$ in $\pi_{1}(F)$.

\section{Proof of Proposition 1}

Proposition 1. Let $\mathscr{U}$ be a surface consisting of a finite union of quadrilaterals. Let $m$ be an integer greater than 1. Suppose that:

(i) the degree of any vertex lying in $\mathscr{U}-\partial \mathscr{U}$ is $k=4(2 m-1)$, and

(ii) any vertex lying in $\partial \mathscr{U}$ has degree less than $k-1$,

then there exists a closed surface consisting of a finite union of quadrilaterals $\bar{u}$, containing $\mathscr{U}$, such that the degree of any vertex is $k$.

Proof. For convenience we orient each edge in $\mathscr{U}$ in such a way that edges of any pair of opposite sides of any quadrilateral have a coherent orientation. All identifications of quadrilaterals along edges will be understood to respect these orientations.

We now make a definition. Let $x$ be a vertex in $\mathbb{H}^{2}$ endowed with its tessellation by quadrilaterals; denote by $Q(x)$ the set of $k$ quadrilaterals sharing $x$. A wedge of quadrilaterals is a union $W$ of $r$ quadrilaterals in $Q(x)$ such that $r<k$ and $W$ is a surface. So in particular $x$ is an ordinary boundary point of $W$. The two edges of $W$ containing $x$ are called the attaching edges of $W$.

We break the proof into several steps:

Step 1. There is a union of quadrilaterals $\mathscr{V}$ containing $\mathscr{U}$, such that the degree of any vertex in $\partial \mathscr{V}$ is 1 or 2 . Moreover, any component of $\partial \mathscr{V}$ consists of an even number of edges.

Proof of Step 1. If there is no vertex of degree greater than 2 on $\partial \mathscr{U}$ and all the boundary components have an even number of edges we are done. If some component, say $C$, does not satisfy these requirements we consider the two cases: $C$ has more than one vertex (Case 1 ) and $C$ has exactly one vertex (Case 2). 


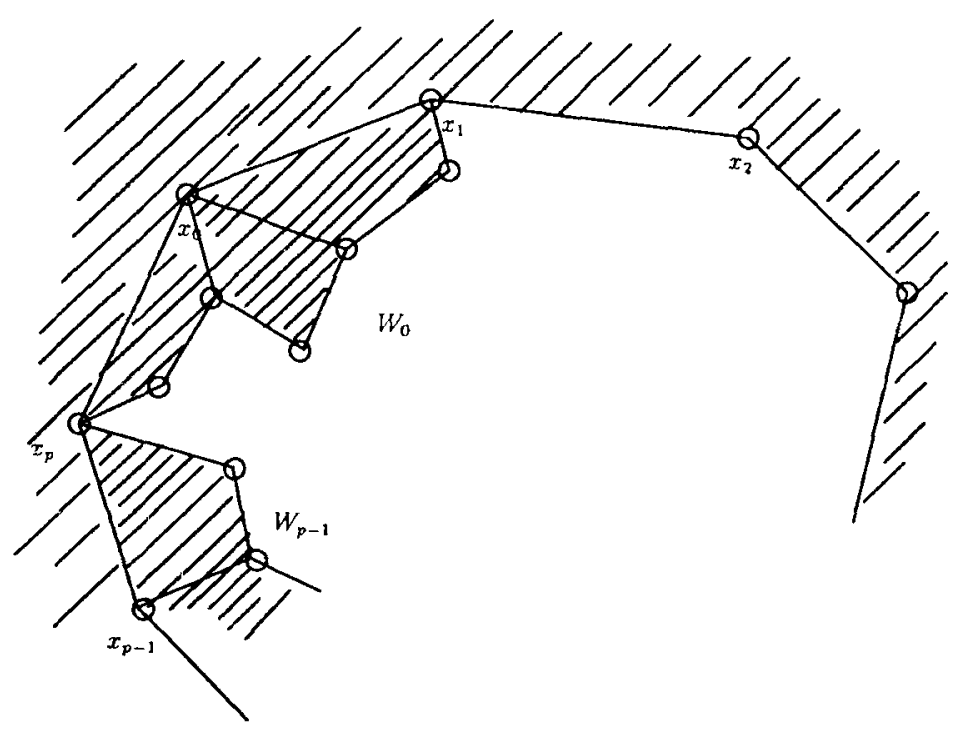

Fig. 4. Case 1.

Case 1: There Is More than One Vertex on $C$ (Fig. 4). We first orient $C$. Then we begin at any vertex $x_{0}$ of $C$ of degree $k-r_{0}$ at least 2 . We call $e$ and $e^{\prime}$ the two edges of $\partial \mathscr{U}$ containing $x_{0}$. We glue to $\mathscr{U}$ a wedge $W_{0}$ of $r_{0}$ quadrilaterals by gluing, edge to edge, the attaching edges of $W_{0}$ to $e \cup e^{\prime}$, so that $x_{0}$ becomes an interior vertex of $\mathscr{U} \cup W_{0}$. We go to the next vertex of $C$ ("next" with respect to $C$ 's orientation), and pursue the operation of gluing wedges until we reach $x_{p}$, the vertex before $x_{0}$ on $C$. Because of the hypothesis on degrees of vertices on $C$ we know that, for all $i, 0<i<p$, the vertex $x_{i}$ has degree at most $k-1$ in $\mathscr{U} \cup W_{0} \cup \cdots \cup W_{i-1}$. This means that after having glued $W_{i}$ there is only one vertex shared by $W_{i-1}$ and $W_{i}$ and it has degree 2 . All the vertices on

$$
\partial\left(\mathscr{U} \cup W_{0} \cup \cdots \cup W_{i}\right)
$$

belonging to only one wedge $W_{j}$ for $0<j<i$ obviously have degrees 1 or 2 .

If $d\left(x_{p}\right)=k-2$ in $\mathscr{U}$, then $d\left(x_{p}\right)=k$ in $\mathscr{U} \cup W_{0} \cup \cdots \cup W_{p-1}$, and we identify the sides of $W_{p-1}$ and $W_{0}$ to be identified in order to turn $x_{p}$ into an interior vertex. Here also the above identification involves a vertex (besides $x_{p}$ ) shared by $W_{p-1}$ and $W_{0}$ and with degree 2 . All the other vertices have degrees equal to either 1 or 2.

If $d\left(x_{p}\right)=d<k-2$ in $\mathscr{U}$, then $d\left(x_{p}\right)=d+2$ in $\mathscr{U} \cup W_{0} \cup \cdots \cup W_{p-1}$, and as usual we can glue a wedge $W_{p}$ of $r_{p}=k-d-2$ quadrilaterals about $x_{p}$. The same conclusion holds for degrees of boundary vertices in this case.

Case 2: There Is Only One Vertex $x_{0}$ on $C$ (Fig. 5). Then there is only one edge, and we glue a quadrilateral $W_{0}$ along this edge. Here again we have two cases:

1. If $d\left(x_{0}\right)=k-2$ in $\mathscr{U}$ we identify the two edges of $W_{0}$ in order to turn $x_{0}$ 


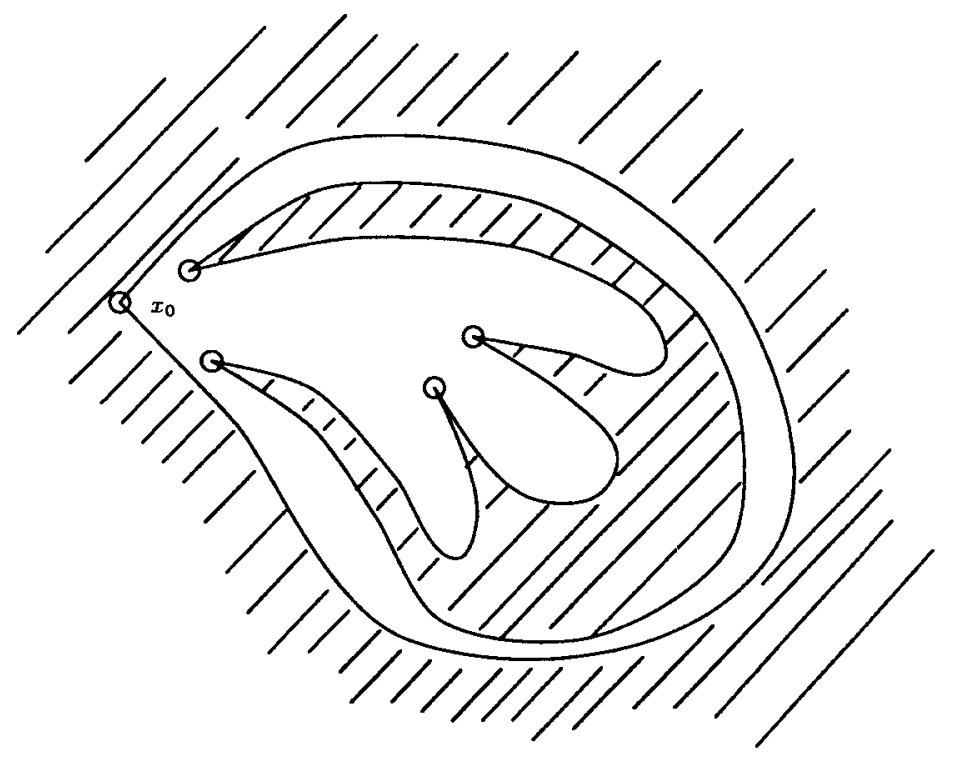

Fig. 5. Case 2 .

into an interior vertex and obtain a boundary component with one vertex of degree 2.

2. If $d\left(x_{0}\right)=d<k-2$ in $\mathscr{U}$ it is $d+2$ in $\mathscr{U} \cup W_{0}$ and we glue a wedge $W_{1}$ of $k-d-2$ quadrilaterals about $x_{0}$.

In each case we obtained the degrees we wanted to obtain.

Let us compute the number of edges.

In Case $1 C$ had $p+1$ vertices, $p>0$, and the surface to consider is

$$
\mathscr{U} \cup W_{0} \cup \cdots \cup W_{q},
$$

where $q=p-1$ or $q=p$ according to whether $x_{p}$ 's degree in $\mathscr{U}$ is equal to $k-2$ or less than $k-2$. We denote by $r_{i}$ the number of quadrilaterals forming $W_{i}$; then the number of free edges coming from the wedges is $\sum_{i=0}^{i=q} 2 r_{i}$, and hence is even.

In Case 2 we consider first the case $d\left(x_{0}\right)=k-2$. We have glued a quadrilateral about $x_{0}$ and obtained a boundary component with one vertex, hence one edge: we again glue a quadrilateral along this edge and obtain a boundary component with three vertices; two of degree 1 and one of degree 4. We apply Case 1 and according to the above calculation we are done. Next we consider the case where $d\left(x_{0}\right)<k-2$ : we have glued a quadrilateral and a wedge $W_{1}$, so we obtain a boundary component with more than one vertex, so we can apply Case 1 here also.

The above can be undergone for each component of $\mathscr{U}$ not satisfying the requirements of Step 1 .

Step 2. There is a union of quadrilaterals $\mathscr{V}^{\prime}$ containing $\mathscr{V}$ such that each boundary component of $\mathscr{V}^{\prime}$ contains exactly two vertices, each having degree equal to either 2 or 4 . 


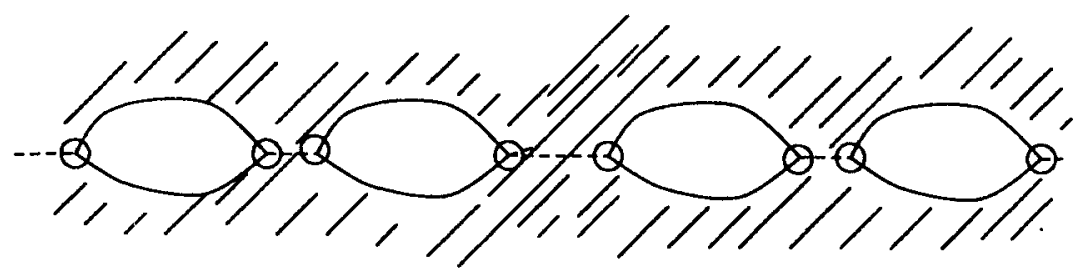

Fig. 6. Step 2.

Proof of Step 2. Take two disjoint copies of $\mathscr{V}, \mathscr{V}_{1}$ and $\mathscr{V}_{2}$, and paste them along part of their boundaries in the following way: identify only one pair of edges out of two; see Fig. 6.

Since any component of $\partial \mathscr{V}$ consists of an even number of edges we can perform this operation. The resulting union of quadrilaterals $\mathscr{V}^{\prime}$ has the desired properties.

Step 3. There is a union of quadrilaterals $\mathscr{W}$ containing $\mathscr{V}^{\prime}$ such that each boundary component contains exactly two vertices, both having the same degree: 2,4 , or 6 .

Proof of Step 3. Here again take two copies of $\mathscr{V}^{\prime}, \mathscr{V}_{1}^{\prime}$ and $\mathscr{V}_{2}^{\prime}$, and change the orientations of $\mathscr{V}_{2}^{\prime}$ 's edges. Then identify $\mathscr{V}_{1}^{\prime}$ and $\mathscr{V}_{2}^{\prime}$ along one edge of each of their corresponding boundary components, but only for those boundary components having vertices of different degrees (according to Step 2 one has to be 2 and the other 4). Since we have reversed orientations of edges, a vertex of degree 2 on $\partial \mathscr{V}_{1}^{\prime}$ will be identified with one of degree 4 on $\partial \mathscr{V}_{2}^{\prime}$, and vice versa. The conclusion follows.

Step 4. The surface $\mathscr{W}$ of Step 3 can be embedded in one satisfying the conditions at the conclusion of Proposition 1.

Proof of Step 4. Write $k=6 t+r$, where $0 \leq r<6$, and bring $t$ copies $\mathscr{W}_{1}$, $\mathscr{W}_{2}, \ldots, \mathscr{W}_{1}$ of the surface $\mathscr{W}$ whose existence is asserted in Step 3 . Notice that, since $k$ is even, $r$ must be one of 0,2 or 4 .

If $r=0$, glue the $t$ copies as follows: each edge having its vertices of degree 6 in a boundary component of $\mathscr{W}_{i}$ is identified to the opposite edge of the corresponding boundary component of $\mathscr{W}_{i+1}$ for $i=1, \ldots, t-1$, and the same is done for $\mathscr{W}_{t}$ and $\mathscr{W}_{1}$. We now take $k / 4$ copies of the resulting surface and do exactly the same thing as above for degree 6 for boundary components having degrees equal to 4 . At this point all degrees are equal to 2 . We now take $k / 2$ copies of this space and repeat the above process. After that we have a union of quadrilaterals as promised in Proposition 1.

If $r=2$, apply the above trick to $\mathscr{W}_{1}, \ldots, \mathscr{W}_{t}$ and the boundary components with degree 6 vertices, but do not identify the edges of $\mathscr{W}_{1}$ and $\mathscr{W}_{t}$. We get a new union of quadrilaterals where each boundary component has two vertices of the same degree: $6 t, 2$, or 4 ; just glue one quadrilateral along each free edge having endpoints of degree $6 t$, and glue together the edges to be glued in order to get interior vertices of degree $k$ there. So the remaining boundary degrees are 2 or 4 and we finish this case as in the case $r=0$. 


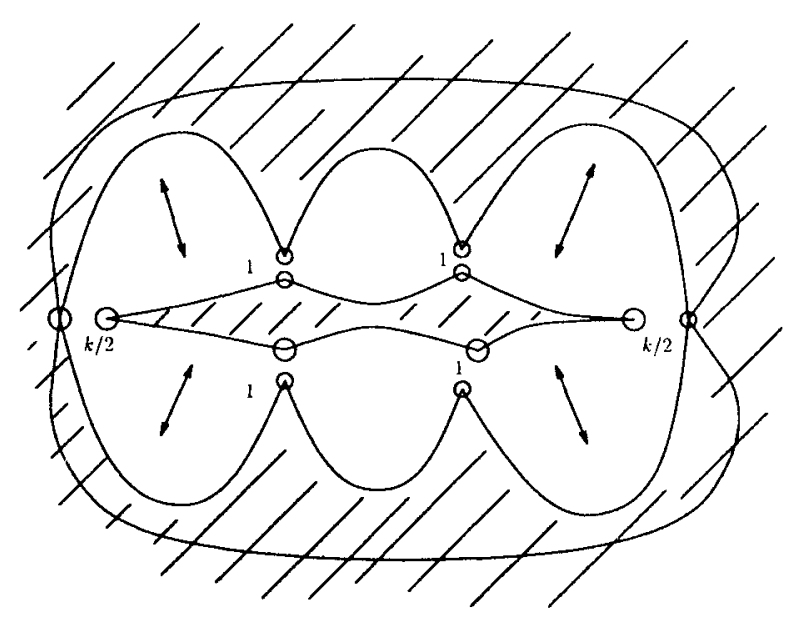

Fig. 7. A surface with six-edge boundary components.

If $r=4$, then $k / 2=6(t / 2)+2$ (recall that $k$ is divisible by 4 , hence $t$ is divisible by 2). This time take $t / 2$ copies of our union of quadrilaterals of Step 4 and apply the same trick again to $\mathscr{W}_{1}, \ldots, \mathscr{W}_{t / 2}$ and the boundary components with degree 6 vertices. We get a surface with two-edge boundary components, and boundary degrees equal to $6 t / 2,2$, or 4 . We add one quadrilateral along each edge having an endpoint of degree $6 t / 2$ (see Fig. 7), and get a new surface with six-edge boundary components and degrees equal either to $6 t / 2+2=k / 2$ or to 1 , and two-edge boundary components and degrees equal to 2 or 4 . It suffices then to take two copies of this surface and to identify edges in such a way that points of degree $k / 2$ are identified together (see Fig. 7).

The result is a union of quadrilaterals having two-edge boundary components and degrees equal to 2 or 4 . We finish this case as in cases $r=0$ and 2.

\section{Acknowledgment}

I wish to thank Professor S. Kojima for all the valuable remarks and simplifications he pointed out to me for this paper.

\section{References}

1. D. Beauquier and M. Nivat, On translating one polyomino to tile the plane, Discrete Comput. Geom. 6 (1991), 575-592.

2. A. M. Brunner, R. G. Burns, and D. Solitar, The subgroup separability of free products of two free groups with cyclic amalgamation, Contemp. Math. 33 (1984), 90-115. 
3. D. B. A. Epstein, J. W. Cannon, D. F. Holt, S. V. F. Levy, M. S. Paterson, and W. P. Thurston, Word Processing in Groups, Jones and Bartlett, Boston, 1992.

4. W. Magnus, Noneuclidean Tessellations and Their Groups, Academic Press, New York, 1974.

5. P. Scott, Subgroups of surface groups are almost geometric, J. London Math. Soc. (2) 17 (1978), $555-565$.

6. P. Scott, Correction to "Subgroups of surface groups are almost geometric", J. London Math. Soc. (2) 32 (1985), 217-220.

Received May 20, 1992, and in revised form November 5, 1992. 